\title{
Moldeo por inyección del acero rápido $\mathrm{M2}^{\left({ }^{(\bullet)}\right.}$
}

\author{
J.M. Ruiz-Román ${ }^{(*)}$, N. Candela ${ }^{(*)}$ y F. Velasco ${ }^{(* *)}$ \\ Resumen Se estudia la viabilidad de la tecnología de Moldeo por Inyección de Polvos (MIM) en la fabricación \\ con acero rápido $\mathrm{M} 2$ a fin de obtener componentes de este material de prestaciones superiores a las \\ que se consiguen mediante métodos convencionales pulvimetalúrgicos. Los ensayos realizados se \\ han dirigido a optimizar los parámetros de la eliminación del ligante y de la sinterización, con objeto \\ de controlar el contenido de carbono y la densidad final de las piezas, así como evitar la aparición de \\ los defectos típicos de esta tecnología.
}

Palabras clave: Moldeo por inyección de polvos (MIM). Pulvimetalurgia. Acero rápido. Ligante. Sinterización.

\section{Injection molding of M2 high speed steel}

\begin{abstract}
The purpose of this work is to study the feasibility of Powder Injection Molding in order to obtain M2 High Speed Steels with higher performances than those obtained by conventional P/M. With this object trials have been carried out in order to optimize all the process steps (mixing, injection, debinding and sintering), and to evaluate the mechanical properties (hardness and transverse tensile strengh) of the manufactured M2 HSS sample.
\end{abstract}

Keywords: Metal injection molding (MIM). Powder metallurgy. High speed steel. Binder. Sintering.

\section{INTRODUCCIÓN}

El Moldeo por Inyección de Polvos (MIM) es un procedimiento pulvimetalúrgico que tuvo sus orígenes a principios de los años veinte (1) y que ha experimentado un gran avance, fundamentalmente, en los últimos diez años en la fabricación de componentes médicos, militares, aeroespaciales, etc. de formas complicadas, con elevadas propiedades y en su forma casi final (2-5). Se estima que el crecimiento anual de los materiales fabricados con este procedimiento es superior al $50 \%$ (6).

El moldeo por inyección (ampliamente utilizado para la obtención de piezas de plástico), como procedimiento para la fabricación de componentes de materiales metálicos o cerámicos, precisa previamente la obtención de mezclas de estos polvos con polímeros termoplásticos para su moldeo por inyec-

$(\bullet$ Trabajo recibido el día 23 de febrero de 1996

(*) Dpto. de Ingeniería de Materiales. E.T.S. de Ingenieros de Minas. Universidad Politécnica de Madrid. c/ Ríos Rosas, 21. 28003-Madrid (España).

(**) Universidad Jaume I. Campus de Penyeta Roja. 12071Castellón (España). ción como si de un plástico se tratara (7). Ello requiere un paso intermedio antes de la sinterización, que ha de permitir eliminar el ligante añadido (8).

El MIM se está mostrando como una tecnología interesante, desde el punto de vista económico, cuando se trata de fabricar componentes metálicos de pequeño tamaño, formas complejas y altas prestaciones, ya que este procedimiento hace innecesarias operaciones secundarias de acabado al proporcionar los productos en su forma final. Esto es lo que hace al MIM especialmente interesante para el caso de componentes fabricados con metal duro, como es el del acero rápido ( 9 y 10) y concretamente para el acero M2, objeto de este estudio (11).

Por tanto, el propósito de este trabajo es estudiar la viabilidad de la tecnología MIM en la fabricación con acero rápido $\mathrm{M} 2$, con objeto de determinar si se pueden obtener componentes de este material de prestaciones superiores a las obtenidas por métodos convencionales, y optimizar los parámetros de la eliminación del ligante y la sinterización para controlar el contenido de carbono y la densidad final de los componentes, así como evitar la aparición de defectos típicos de esta tecnología. 


\section{PROCEDIMIENTO EXPERIMENTAL}

Se partió de un polvo de acero rápido M2 atomizado por gas (cuya composición química puede verse en la tabla I) de tamaño medio de partícula de $22 \mu \mathrm{m}$.

El ligante utilizado es una mezcla de ceras poliolefínicas suministrado por la casa Höescht cuyo nombre comercial es Hostamont TP EK 583.

La mezcla se llevó a cabo en un mezclador a $140{ }^{\circ} \mathrm{C}$, durante $4 \mathrm{~h}$, y en la proporción siguiente:

- $63 \%$ en volumen de polvo de M2

- $37 \%$ en volumen de ligante

La mezcla, en peso, presentó las siguientes proporciones:

- $93 \%$ de polvo de M2

- $7 \%$ de ligante

Una vez homogeneizada la mezcla, se procedió al granulado de la misma en una trituradora de impactos.

La inyección se llevó a cabo en un inyector de la casa Arburg, modelo Allrounder 370C, a una presión de $87,5 \mathrm{MPa}$, obteniéndose probetas de tracción de $150 \mathrm{~mm} \times 20 \mathrm{~mm} \times 1,5 \mathrm{~mm}$.

La eliminación del ligante se realizó en dos etapas diferentes:

- Extracción con disolventes

Esta primera fase de eliminación del ligante se llevó a cabo siguiendo el programa indicado en la tabla II.

- Eliminación térmica

Posteriormente, y tras una serie de ensayos para obtener la temperatura idónea de eliminación del ligante y reducir al menor tiempo posible la permanencia de las probetas en el horno, se sometió a las mismas, en atmósfera de argón, al ciclo de temperaturas que se indica en la tabla III.

Para seleccionar la temperatura de sinterización del material inyectado se realizaron ensayos dilatométricos, como el que muestra la figura 1 , y de sinterabilidad, donde se puede apreciar que la densificación del mismo se produce para una temperatura
Tabla II.- Condiciones de eliminación del ligante para su extracción con disolventes

TABLE II.- Solvent extraction debinding conditions

\begin{tabular}{|c|c|}
\hline Disolvente & Acetona \\
\hline Temperatura, ${ }^{\circ} \mathrm{C}$ & 65 \\
\hline Tiempo, $\mathrm{h}$ & 7 \\
\hline \% eliminado & $50-55$ \\
\hline
\end{tabular}

TABLA III.- Ciclo de eliminación térmica del ligante

TABLE III.- Thermal debinding cycle

\begin{tabular}{|c|c|}
\hline Temperatura, ${ }^{\circ} \mathrm{C}$ & Tiempo, min \\
\hline $0-140$ & 60 \\
$140-190$ & 100 \\
$190-205$ & 180 \\
$205-350$ & 250 \\
350 & 30 \\
$350-\mathrm{RT}$ & 120 \\
\hline
\end{tabular}

aproximada de $1.150{ }^{\circ} \mathrm{C}$. El ciclo de sinterización seguido para el acero rápido M2 se observa en la tabla IV.

Las probetas sinterizadas fueron austenizadas en atmósfera de argón (para evitar su oxidación), a una

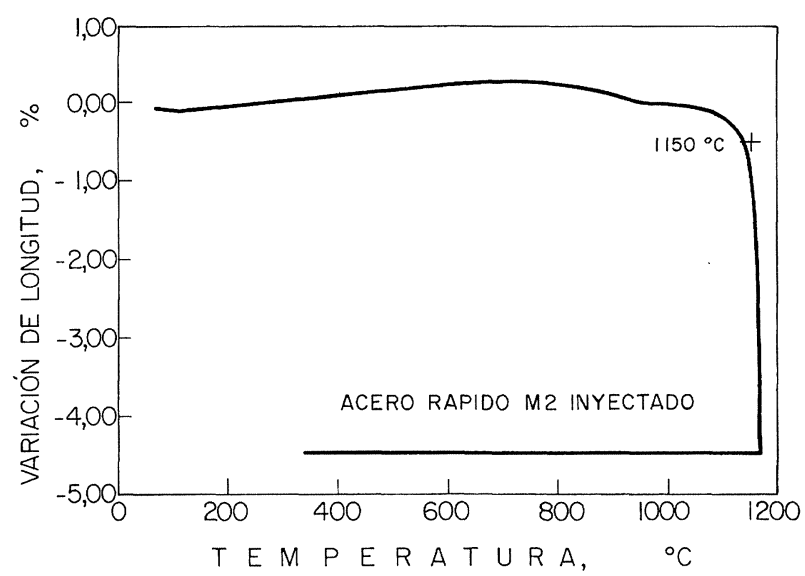

FIG. 1.- Curva dilatométrica del acero rápido M2 inyectado.

FIG. 1.- Dilatometric run for HSS M2 injection moulded.

Tabla I.- Composición química del polvo de acero rápido M2 de la firma Osprey, para moldeo por inyección, en \%

TABLE I.- Chemical composition of Osprey M2 high speed steel powder, in \%

\begin{tabular}{|c|c|c|c|c|c|c|c|c|}
\hline Fe & Co & Cr & W & C & Si & Mo & V & Mn \\
\hline Base & 0,55 & 4,27 & 6,63 & 0,9 & 0,4 & 5,31 & 2,17 & 0,4 \\
\hline
\end{tabular}


TABLA IV.- Ciclo de sinterización para el acero rápido M2 fabricado por tecnología MIM

TABLE IV.- Sintering temperature cycle of an injection molded M2 high speed steel

\begin{tabular}{|c|c|c|}
\hline $\mathrm{T},{ }^{\circ} \mathrm{C}$ & Vel. cal., ${ }^{\circ} \mathrm{C} / \mathrm{min}$ & $t, \mathrm{~min}$ \\
\hline $25-205$ & 2 & 90 \\
205 & - & 15 \\
$205-390$ & 2 & 92 \\
390 & - & 15 \\
$390-1.150$ & 5 & 152 \\
1.150 & - & 30 \\
$1.150-\mathrm{RT}$ & - & - \\
\hline
\end{tabular}

temperatura de $1.200{ }^{\circ} \mathrm{C}$ durante $15 \mathrm{~min}$, y enfriadas en aceite.

Para efectuar el revenido del material inyectado, se realizó un estudio dilatométrico con objeto de determinar la temperatura adecuada y el número de revenidos necesarios.

\section{RESULTADOS Y DISCUSIÓN}

\subsection{Eliminación del ligante y sinterización}

Con el ciclo completo de eliminación del ligante anteriormente expuesto, se elimina aproximadamente un $77 \%$ del mismo al término de las dos etapas, como se aprecia en la figura 2. Esto

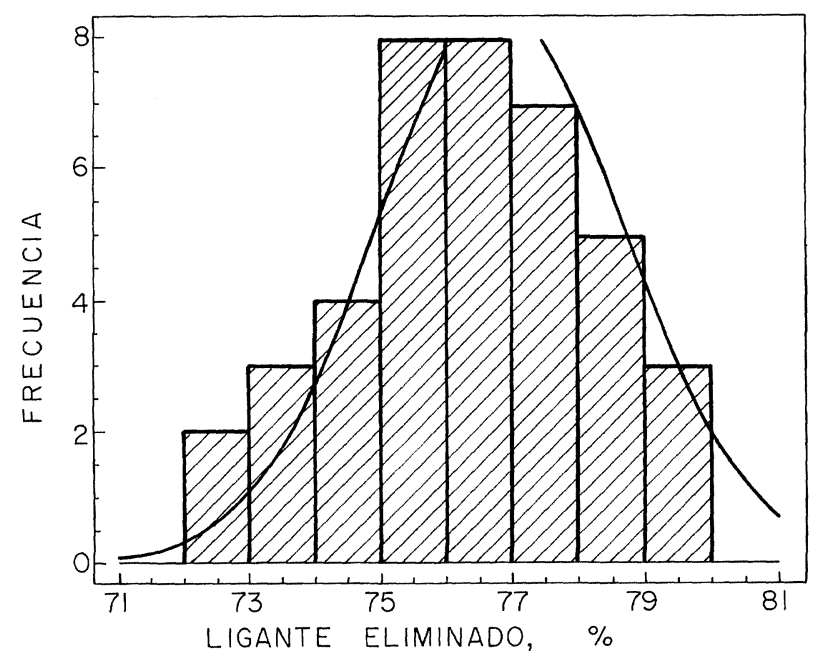

FIG. 2.- Histograma de frecuencias del proceso de eliminación del ligante.

FIG. 2.- Frecuency histogram of debinding process. quiere decir que cuando las probetas entren en el horno de sinterización todavía contendrán del orden de un $22 \%$ de ligante.

Este contenido de ligante va a permitir trabajar con una temperatura de sinterización de $1.150{ }^{\circ} \mathrm{C}$, que viene a ser del orden de $100{ }^{\circ} \mathrm{C}$ menor que la necesaria para sinterizar un acero rápido $\mathrm{M} 2$ fabricado por vía pulvimetalúrgica convencional.

Pero, por el contrario, ese contenido de ligante que todavía presentan los compactos en marrón ha de ser eliminado durante la sinterización, lo que dificulta esta etapa.

Este ligante sería eliminado progresivamente durante las primeras etapas de la sinterización y sería arrastrado por la corriente de gas $\left(\mathrm{N}_{2}-\mathrm{H}_{2}\right)$ que forma la atmósfera de sinterización, y expulsado al exterior. Pero esto no sucedía así, sino que parte del ligante se expulsaba al exterior, pero otra parte se quedaba dentro de la cámara del horno $\mathrm{y}$, al enfriar, se depositaba en la cara superior del material, produciendo unas carburaciones tan grandes que daban lugar a fusiones, con lo que el resultado era la obtención de una fundición en lugar de un acero rápido, como se aprecia en la figura 3 , en la que se muestra el gradiente de concentración de carbono desde la periferia (Fig. 3a, donde se aprecia una microestructura sobrecarburada con grandes áreas eutécticas) al centro (Fig. 3b, donde se aprecia la microestructura óptima de un acero rápido).

Para evitar esto, se procedió a introducir el material en un lecho de alúmina, de tal manera que el ligante, en vez de expulsarse a la atmósfera del horno durante las primeras rampas de calentamiento del ciclo de sinterización, fuese absorbido por el lecho y quedase retenido en él, evitándose su deposición posterior sobre las probetas en un proceso de wick debinding o eliminación del ligante por capilaridad en lecho poroso.

Los ensayos de sinterabilidad dieron como temperatura óptima de sinterización $1.150{ }^{\circ} \mathrm{C}$, ya que a partir de esta temperatura se consigue una densificación completa y una correcta microestructura, como se aprecia en la figura $3 b$, en la que se observa una matriz ferrítica repleta de finos carburos de pequeño tamaño homgéneamente distribuidos. Una vez determinada la temperatura de sinterización, el siguiente paso es optimizar el ciclo de temperaturas, ya que el ligante presente en el compacto debe eliminarse, de manera lenta y progresiva, durante las primeras etapas de la sinterización. Si este compacto se intentara sinterizar a una velocidad de calentamiento normal de $10{ }^{\circ} \mathrm{C} / \mathrm{min}$, el material se hincharía con el consiguiente agrietamiento del mismo, como se aprecia en la figura 4. Esto se debe a la gran energía superficial del material, la superficie de la probeta sinteriza más deprisa que el interior, sobrepasándose el segundo estado de la sinterización en la periferia cuando en el interior no se ha alcanzado. Esto 

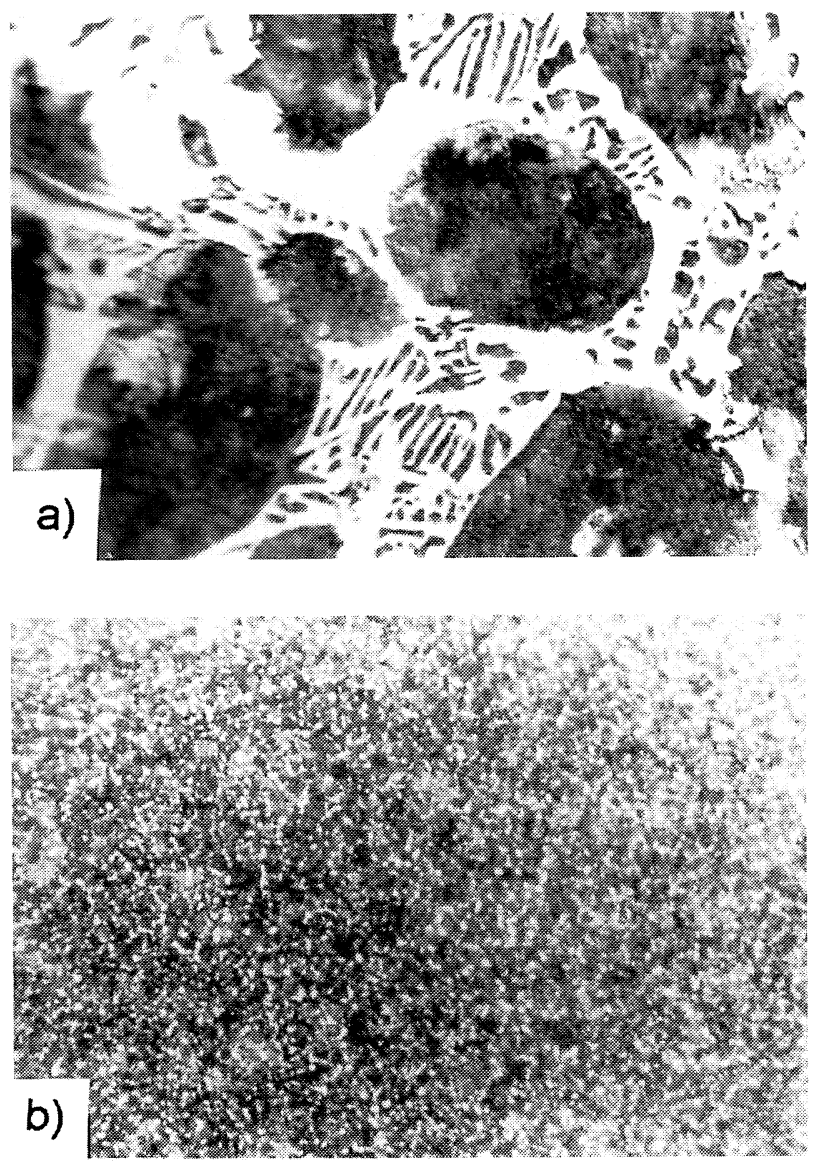

FIG. 3.- Microestructura del acero rápido M2 sinterizado a $1.150{ }^{\circ} \mathrm{C}$. a) En atmósfera de $\mathrm{N}_{2}-\mathrm{H}_{2}$. $\times 250$. b) En lecho de $\mathrm{Al}_{2} \mathrm{O}_{3} . \times 250$.

FIG. 3.- M2 high speed steel injection molded, sintered at $1,150{ }^{\circ} \mathrm{C}$. a) In $\mathrm{N}_{2}-\mathrm{H}_{2}$ atmosphere. $\times 250$. b) With $\mathrm{Al}_{2} \mathrm{O}_{3}$ wick debinding. $\times 250$.

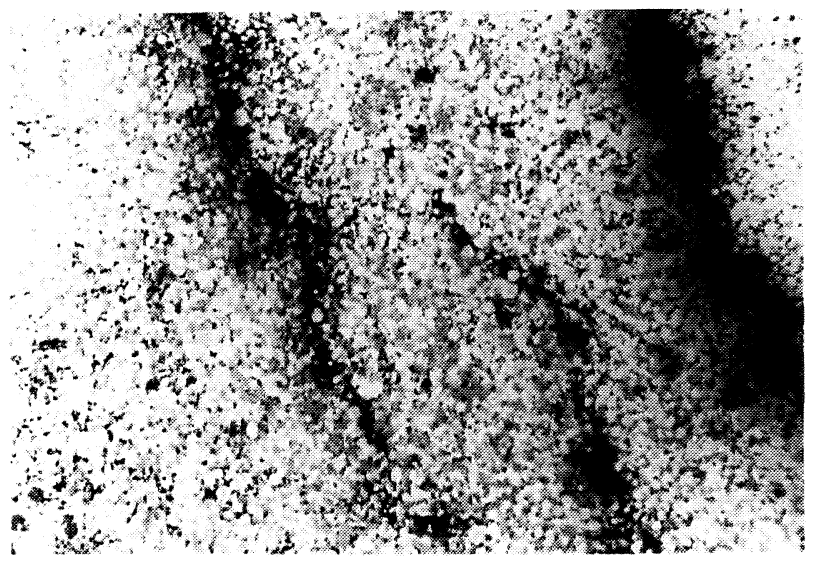

FIG. 4.- Microestructura del acero rápido M2 inyectado y sinterizado a $1.150{ }^{\circ} \mathrm{C}$, a una velocidad de calentamiento de $10{ }^{\circ} \mathrm{C} / \mathrm{min} . \times 250$.

FIG. 4.- Injection molded M2 high speed steel sintered at $1,150{ }^{\circ} \mathrm{C}$ with $10{ }^{\circ} \mathrm{C} / \mathrm{min}$ heating rate. $\times 250$. implica que la porosidad de las zonas externas de la probeta será cerrada, o lo que es lo mismo, que los canales de salida del ligante se habrán cerrado impidiendo su escape, lo que genera un estado tensional tan grande que llega a romper el material.

Para evitar esto, se tiene que lograr que no se produzca un gradiente de temperaturas importante entre la periferia y el núcleo de las probetas, con objeto de conseguir que todo el material sinterice homogéneamente $y$, además, lo suficientemente despacio para que durante las primeras etapas de la sinterización se pueda eliminar todo el ligante remanente; por este motivo, se estableció el ciclo de sinterización expuesto anteriormente.

\subsection{Tratamientos térmicos}

Como se aprecia en la figura 5, el primer ensayo dilatométrico (curva a) indicó como temperatura del primer revenido $580{ }^{\circ} \mathrm{C}$, que es a la que se aprecia que se ha producido transformación martensítica. Ese acero templado y revenido se sometió a otro estudio dilatométrico (curva b), en el que se pudo comprobar que no se obtendría ninguna mejora con un segundo revenido. Esto significa que la austenita retenida en el tratamiento de temple se ha transformado en su totalidad en martensita durante el primer revenido.

Lo anteriormente expuesto era previsible, ya que la temperatura de temple, por limitaciones de los equipos disponibles, era insuficiente para disolver en la austenita la máxima cantidad posible de elementos de aleación, lo que originaría austenitas más estables que requerirían dos o más revenidos, como se recomienda en los aceros de este tipo obtenidos por los procedimientos convencionales de fusión, colada y forja.

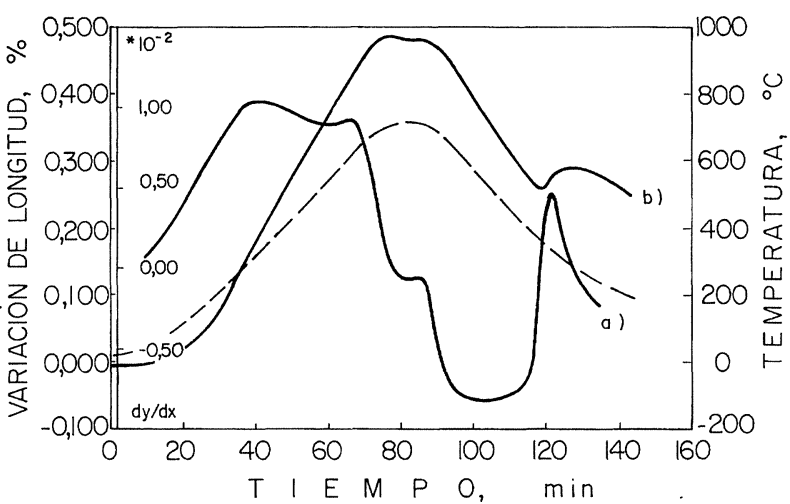

FIG. 5.- Dilatometría del acero rápido M2 templado en aceite a una temperatura de austenización de $1.200{ }^{\circ} \mathrm{C}$.

FIG. 5.-Dilatometric run of an injection molded M2 high speed steel oil quenched at $1,200{ }^{\circ} \mathrm{C}$. 


\subsection{Propiedades mecánicas}

Las figuras 6 y 7 muestran los resultados de dureza obtenidos con el acero rápido $\mathrm{M} 2$ inyectado tanto en su estado sinterizado como en el de temple y revenido. Dichos resultados se tomaron en la escala Rockwell A para poder comparar el lógico aumento de la dureza entre ambos estados.

En cuanto a la resistencia a flexión, en la figura 8 se aprecian los resultados obtenidos para el acero M2 inyectado en su estado de sinterizado, que son los menores que se pueden conseguir con este material a causa de su estado microestructural.

Estos resultados muestran las grandes prestaciones que se pueden obtener con este tipo de aceros inyectados, debido tanto a la presencia de superfinos nitruros precipitados, como al pequeño tamaño, y a la homogénea distribución de los carburos, si bien la presencia de estos nitruros, causada por el empleo de la atmósfera de $\mathrm{N}_{2}-\mathrm{H}_{2}$, debe ser investigada con mayor profundidad.

\section{CONCLUSIONES}

Con el objetivo de analizar la posibilidad de utilizar el moldeo por inyección de polvos como una tecnología con la que conseguir aceros rápidos de propiedades superiores a los fabricados con tecnología convencional, se han estudiado diferentes variables de los procesos de eliminación del ligante, sinterización y tratamientos térmicos, que han llevado a las siguientes conclusiones:

- Desde una correcta mezcla, inyección, eliminación del ligante y sinterización, se pueden obte-

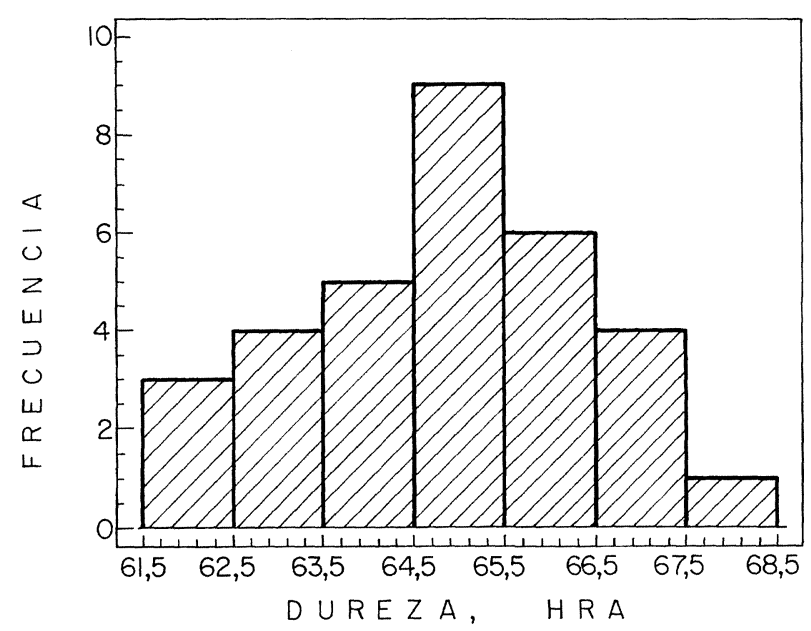

FIG. 6.- Histograma de frecuencias de la dureza del acero rápido $\mathrm{M} 2$ sinterizado a $1.150{ }^{\circ} \mathrm{C}$.

FIG. 6.- Hardness frequency histogram of an injection molded M2 high speed steel sintered at $1,150{ }^{\circ} \mathrm{C}$.

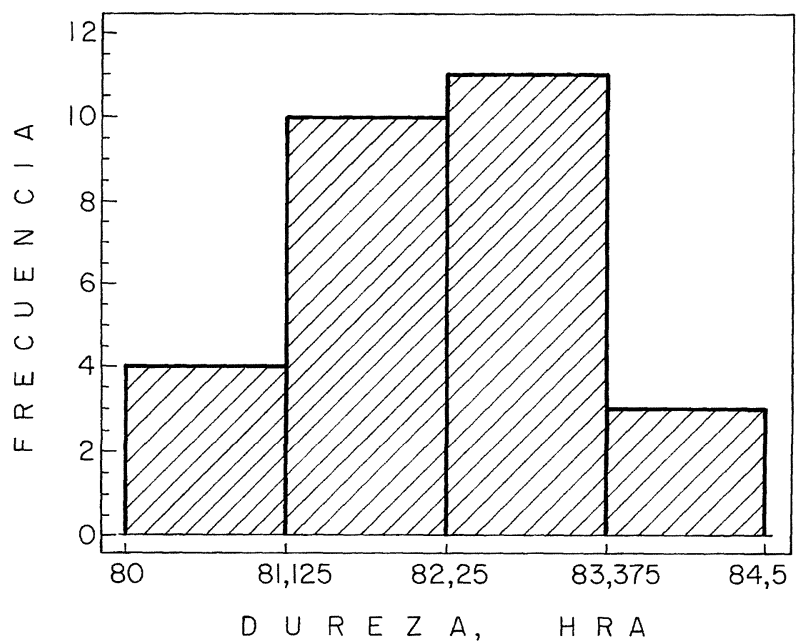

FIG. 7.- Histograma de frecuencias de dureza del acero rápido $\mathrm{M} 2$ templado y revenido a $580{ }^{\circ} \mathrm{C}$.

FIG. 7.- Hardness frequency histogram of an injection molded M2 high speed steel tempered at $580{ }^{\circ} \mathrm{C}$.

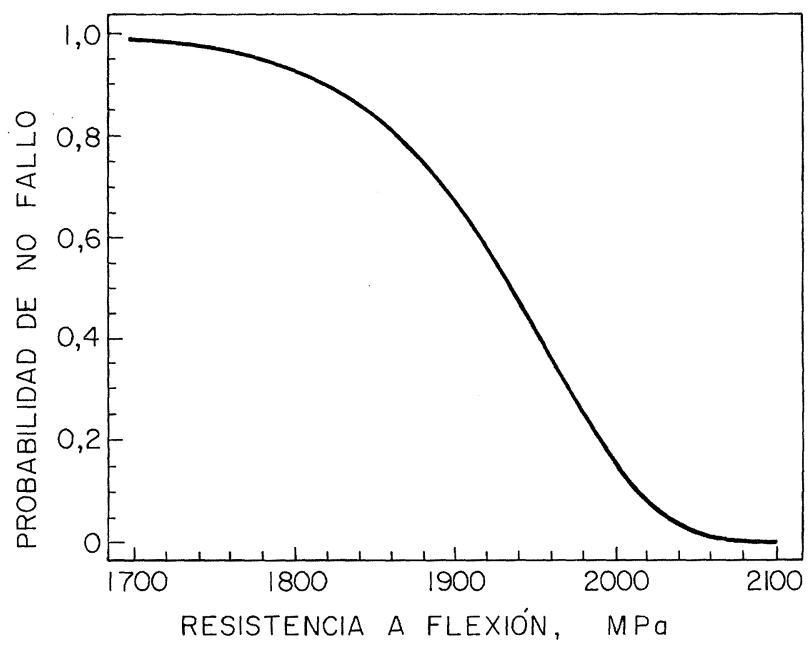

FIG. 8.- Función de supervivencia de la resistencia a flexión del acero rápido $\mathrm{M} 2$ sinterizado a $1.150^{\circ} \mathrm{C}$.

FIG. 8.- Tensile strengh survivor function of an injection molded M2 high speed steel sintered at $1,150^{\circ} \mathrm{C}$.

ner estructuras con carburos finos uniformemente distribuidos en la matriz que permiten alcanzar elevados valores tanto de las propiedades físicas como de las mecánicas en el acero rápido M2 inyectado, como pueden ser un valor de dureza de 65 HRA y $2.000 \mathrm{MPa}$ de resistencia a flexión en estado sinterizado.

- El conjunto de una eliminación del ligante con disolventes y una posterior eliminación térmica se ha mostrado como un proceso idóneo no sólo para eliminar el ligante sino para evitar distorsiones y defectos en el material. 
- Una presencia de un $20 \%$ de ligante al comienzo de la sinterización permite llevarla a cabo a $1.150{ }^{\circ} \mathrm{C}$, consiguiéndose una completa densificación y una microestructura y propiedades óptimas.

- La sinterización en lecho de alúmina se ha mostrado como un método correcto para la eliminación total del ligante, para el control del carbono en las piezas y para evitar la aparición de distorsiones en las mismas, haciendo menos necesario el control de la atmósfera del horno.

\section{REFERENCIAS}

(1) German, R.M., Hens, K.F. y Lin, S.P. Ceram. Bull., 70 (8), 1991: 1.294.

(2) Sumitomo introduces Metamold Metal Injection Moulding. Metal Powder Report. Nov. 1988: 776.

(3) German, R.M. Powder Metall. Int., 25 (4), 1993: 165.
(4) Ruiz-Roman, J.M., Velasco, F. y Cambronero, L.E.G. Moldeo por Inyección de Polvos. Estado Actual y Perspectivas de Futuro. IX Congreso Intern. de Minería y Metalurgia. León 24-28 mayo 1994.

(5) Hens, K.F., Lee, D., Lin, S.P. y German, R.M. Powder Metall. Int., 23 (1), 1991: 15.

(6) P/M'89: Special Report on Progress in Injection Moulding. Powder Metall. Int., 21 (5), 1989: 37.

(7) ERICKSON, A.R. y WIECH, R.E. Injection Molding. Metals Handbook. ASM. Vol. 7, p. 495.

(8) Gummeson, P.U. Int. J. Powder Metall., 25 (3), 1989: 207.

(9) Miura, H., Gondo, H., Honda, T. y Kono, T. Carbon and Structure Control of Injection Molded High Speed Steels. Proc. 1993 Powder Metallurgy World Cong. 1993: 273-276.

(10) Sumitomo. Powder Metall. Int., Nov. 1988.

(11) Ruiz-Roman, J.M. "La tecnología MIM aplicada a la obtención del acero rápido M2. Comparación con el proceso pulvimetalúrgico convencional”. Tesis Doctoral. Dpto. de Ingeniería de Materiales. E.T.S. de Ingenieros de Minas. U.P.M., 1995. 